\title{
Prediction and In Silico Identification of Novel B-Cells and T-Cells Epitopes in the S1-Spike Glycoprotein of M41 and CR88 (793/B) Infectious Bronchitis Virus Serotypes for Application in Peptide Vaccines
}

\author{
Faruku Bande,, ${ }^{1,2}$ Siti Suri Arshad, ${ }^{1}$ Mohd Hair Bejo, ${ }^{1,3}$ Saeid Kadkhodaei, \\ and Abdul Rahman Omar ${ }^{1,3}$ \\ ${ }^{1}$ Department of Veterinary Pathology and Microbiology, Faculty of Veterinary Medicine, Universiti Putra Malaysia, \\ 43400 Serdang, Selangor Darul Ehsan, Malaysia \\ ${ }^{2}$ Department of Veterinary Services, Ministry of Animal Health and Fisheries Development, PMB 2109, \\ Usman Faruk Secretariat, Sokoto State, Nigeria \\ ${ }^{3}$ Laboratory of Vaccine and Immunotherapeutic, Institute of Bioscience, Universiti Putra Malaysia, 43400 Serdang, \\ Selangor Darul Ehsan, Malaysia \\ ${ }^{4}$ Institute of Tropical Agriculture, Universiti Putra Malaysia, 43400 Serdang, Malaysia
}

Correspondence should be addressed to Faruku Bande; bandeyabo@yahoo.com and Siti Suri Arshad; suri@upm.edu.my

Received 8 March 2016; Revised 30 May 2016; Accepted 1 August 2016

Academic Editor: Rita Casadio

Copyright (C) 2016 Faruku Bande et al. This is an open access article distributed under the Creative Commons Attribution License, which permits unrestricted use, distribution, and reproduction in any medium, provided the original work is properly cited.

\begin{abstract}
Bioinformatic analysis was used to predict antigenic B-cell and T-cell epitopes within the S1 glycoprotein of M41 and CR88 IBV strains. A conserved linear B-cell epitope peptide, YTSNETTDVTS ${ }^{175-185}$, was identified in M41 IBV strains while three such epitopes types namely, VSNASPNSGGVD ${ }^{279-290}$, HPKCNFRPENI ${ }^{328-338}$, and NETNNAGSVSDCTAGT ${ }^{54-69}$, were predicted in CR88 IBV strains. Analysis of MHCI binding peptides in M41 IBV strains revealed the presence of 15 antigenic peptides out of which 12 were highly conserved in $96-100 \%$ of the total M41 strains analysed. Interestingly three of these peptides, GGPITYKVM ${ }^{208}$, WFNSLSVSI $^{356}$, and YLADAGLAI ${ }^{472}$, relatively had high antigenicity index $(>1.0)$. On the other hand, 11 MHCI binding epitope peptides were identified in CR88 IBV strains. Of these, five peptides were found to be highly conserved with a range between $90 \%$ and $97 \%$. However, WFNSLSVSL ${ }^{358}$, SYNISAASV ${ }^{88}$, and YNISAASVA ${ }^{89}$ peptides comparably showed high antigenicity scores $(>1.0)$. Combination of antigenic B-cells and T-cells peptides that are conserved across many strains as approach to evoke humoral and CTL immune response will potentially lead to a broad-based vaccine that could reduce the challenges in using live attenuated vaccine technology in the control of IBV infection in poultry.
\end{abstract}

\section{Introduction}

Infectious bronchitis virus (IBV) is a single stranded, enveloped RNA virus belonging to the family Coronaviridae, order Nidovirales [1]. The virus causes infectious bronchitis (IB), a contagious disease associated with huge economic loses in poultry industry worldwide [2]. Of major concern in the control of IB is the continued emergence of variant IBV strains that differ in terms of their tissue tropism, pathogenicity, and cross protection. Over the years, serological and molecular studies have been carried out extensively to determine the epidemiology of local IBV strains. Remarkably, both classical and variants IBV strains have been reported in different countries [3]. Among the widely identified IBV strains are IBV M41 (classical strain), originally recognized in USA $[4,5]$, and CR88 (variant strain otherwise known as $793 / \mathrm{B}$ or $4 / 91$ ) which was first reported in Europe [3, 6, 7].

Over the years, control of IBV infection largely depends on vaccination using live attenuated and killed vaccines. 
However, one of the challenges with live attenuated IB vaccines is that such vaccines are reported to encourage mutation and recombination, thus leading to the emergence of new variant strains. Live attenuated vaccines have also been linked with reversion to virulence, severe disease, and increased mortality rate [8-10]. On the other hand, killed vaccines induce humoral but not cell mediated immune (CMI) response and in most cases require adjuvants as well as repeated boosting especially in laying chickens and breeder flocks. These challenges therefore necessitate the needs for novel broad vaccines for the control of IB in poultry [11]. To achieve this, stimulation of both humoral (B-cell) and cell mediated immune (CMI) responses is considered very essential for any candidate vaccine [12]. Neutralizing antibodies are important in removing freely circulating IB virus, whereas cytotoxic T- lymphocytes (CTL) response is crucial for the control and clearance of virally infected cells. The latter is achieved through MHCI immune surveillance as well as antigen presentations which is the function of MHCI molecule and both have been associated with the epitope within the S1 glycoprotein [13, 14]. While much has been documented on MHC restricted allele in human and mouse models, little information is available on the biological functions of these molecules in poultry [15]. A large binding groove of $\mathrm{BF} 2 * 2101 \mathrm{MHCI}$ molecule identified in B21 chicken line is thought to confer conformational flexibility to the crucial Arg9 residue which allows remodeling of key peptide-binding sites and play a role in the resistance against poultry viral infections [16]. Chicken MHC B-F molecules have been structurally and functionally linked to mammalian MHC class I molecules and involved in antigen presentation to the $\mathrm{CD}^{+} \mathrm{T}$ lymphocytes, which is crucial in antiviral immune response [17]. Interestingly, the S1 glycoprotein of IBV (520 aa) contains different immune epitopes responsible for both antibodies and CTL-based immune responses, thus playing major protective role as viral antigenic determinant [18].

Currently, the use of peptide based DNA vaccines represents a novel strategy for addressing challenges associated with the control of viral infections $[19,20]$. This technology may employ the use of in silico analysis to predict novel B-cells and T-cells immune epitopes for further use in their in vivo applications. One of the innovations in using this technology is the ability to incorporate several epitope peptides directed against different viruses and/or multiple virus strains into one single delivery system with the view to induce broad and specific immune response in single administration [11]. To date, only few epitope based peptide vaccines have been developed and evaluated against IB [20, 21]. The objective of this study therefore is to identify novel B-cells and T-cell epitopes within the S1 glycoprotein of M41 and CR88 IBV strains. The antigenicity of the predicted peptides was also evaluated.

\section{Materials and Methods}

2.1. Nucleotide Sequence Retrieval. Nucleotide sequences containing complete S1-gene of homologous to M41 and
CR88 IBV strains were retrieved from NCBI database and translated into deduced amino acid sequences for further analysis. Consensus amino acid sequences from the two datasets (M41 and CR88) were used to predict B- and Tcells epitope peptides within S1 glycoprotein. The criteria used for the selection of M41 and CR88 were simply based on epidemiological relevance of the two strains in terms of their wide geographic distribution as classical and variant IBV strains, respectively. All sequences were assembled with Geneious $^{\circledR}$ software R7 version [22]. The names and accession numbers of IBV sequences used in this study are presented in Supplementary File 1 in Supplementary Material available online at http://dx.doi.org/10.1155/2016/5484972.

2.2. Prediction of B-Cell Epitope. Epitopes associated with Bcells were predicted using the BepiPred epitope prediction server version 1.0 [23]. Similarly, antigenicity index of the predicted epitopes was analysed using VaxiJen v2.0 online antigen prediction tool http://www.ddg-pharmfac.net/vaxijen/VaxiJen/VaxiJen.html [22]. Prediction criteria were set at $75 \%$ classifier specificity and 20 epitope lengths with an overlap. The location of the predicted epitope within transmembrane regions was evaluated using TMHMM [24]. Only epitope present at the surface of the membrane was selected and further analysed for antigenicity. For this search, target organism was narrowed to viruses and only scores above 0.4 thresholds were considered as a good antigenic epitope. Further, conservancy analysis was carried out using IEDB tools available http://tools.immuneepitope.org/tools/ conservancy/iedb_input [25].

2.3. Prediction of T-Cell Epitopes. The T-cell epitope prediction was carried out using ProPred-1 software which covers 47 MHC class-I alleles. Both proteasome and immuneproteasome were set at $5 \%$ threshold by MHC class I and only peptides with proteosomal cleavage site at the $\mathrm{C}$ terminus were considered [26].

2.4. Epitope Conservancy Analysis. Immune epitopes were analysed for conservancy and variability using IEDB conservation analysis tool: http://tools.immuneepitope.org/tools/ conservancy/iedb_input [25].

\section{Results}

3.1. Nucleotide Sequence Retrieval. A total of $86 \mathrm{M} 41$ and 72 CR88 IBV isolates were considered in this study. However, to build the consensus sequence, one isolate from each data set was removed due to some unidentified amino acid at one or two positions. The M41 IBV isolates were found to have an average nucleotides sequence length of 1614 (537 aa) while CR88 isolates had an average length of 1653 (553 aa).

3.2. B-Cell Epitope Prediction and Conservancy Analysis. Analysis of M41 associated linear B-cell epitopes revealed the presence of six unique B-cells peptides within the S1 glycoprotein. Of these, only YTSNETTDVTS ${ }^{175-185}$ peptide was found to be antigenic (Table 1). 
TABle 1: Predicted antigenic B-cells linear epitopes within the S1 glycoprotein sequence of M41 IBV strains.

\begin{tabular}{lccc}
\hline Position & Peptides & Length & Antigenicity \\
\hline S33-44 & AFRPGPGWHL & 10 & 0.1545 \\
S52-66 & SSESNNAGSSSGCT & 14 & 0.0842 \\
S83-92 & MTAPSSGMAW & 10 & 0.0728 \\
S175-185 & YTSNETTDVTS & 11 & 0.6727 \\
S403-421 & KSGGSRIQTATEPPVITQH & 19 & 0.2319 \\
\hline
\end{tabular}

Peptides in italic represent antigenic epitope considered.

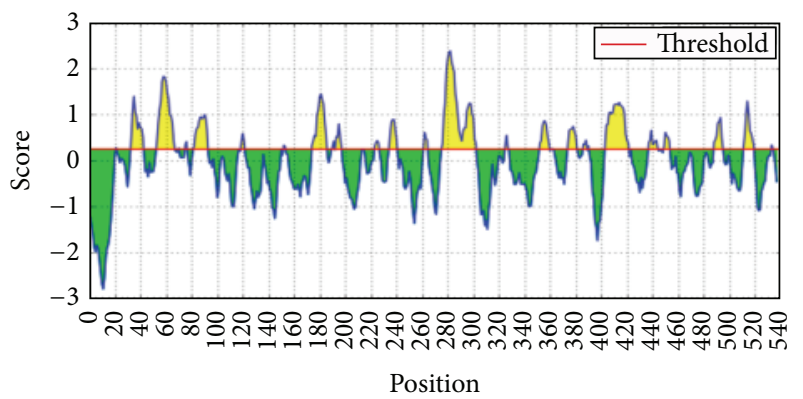

Figure 1: Predicted linear B-cells epitopes and their locations to the surface membrane (yellow) in M41 IBV strains. Note: maximum transmembrane interaction was observed at a threshold of 2.4 within amino acid positions 280 to 290 .

TABLE 2: Predicted antigenic B-cells linear epitopes found in the S1 glycoprotein sequence of CR88 IBV strains.

\begin{tabular}{lccc}
\hline Epitope position & Peptides & Length & Antigenicity \\
\hline S33-44 & AFRPGPGWHL & 10 & 0.1545 \\
S54-69 & NETNNAGSVSDCTAGT & 16 & 0.4237 \\
S83-92 & MTVPPNGMSW & 10 & 0.0306 \\
S279-290 & VSNASPNSGGVD & 12 & 0.7396 \\
S328-338 & HPKCNFRPENI & 11 & 1.9437 \\
S405-414 & KSDGSRIQTR & 10 & 0.3178 \\
\hline
\end{tabular}

Peptides in italic represent antigenic epitope.

Analysis of transmembrane potentials within the M41 IBV S1 gene showed that the predicted linear B-cell epitopes interact with the outside surface membrane especially with maximum threshold occurring at amino acid positions 280 to 290 (Figure 1).

Interestingly, conservancy analysis showed that YTSNETTDVTS ${ }^{175-185}$ peptide is common in $82.35 \%$ of the M41 strains. On the other hand, a total of six antigenic epitope peptides were predicted in CR88 IBV strains; however, three of these peptides, NETNNAGSVSDCTAGT ${ }^{54-69}$, VSNASPNSGGVD ${ }^{279-290}$, and HPKCNFRPENI ${ }^{328-338}$ were demonstrated to be antigenic (Table 2). Further, conservancy analysis showed that the three CR88 associated antigenic B-cell peptides had $39.44 \%, 84.5 \%$, and $43.66 \%$ conservancy rates, respectively.

As with the M41 IBV strains, most of these epitopes are shown to interact with the surface at varying threshold (yellow) as depicted in Figure 2.

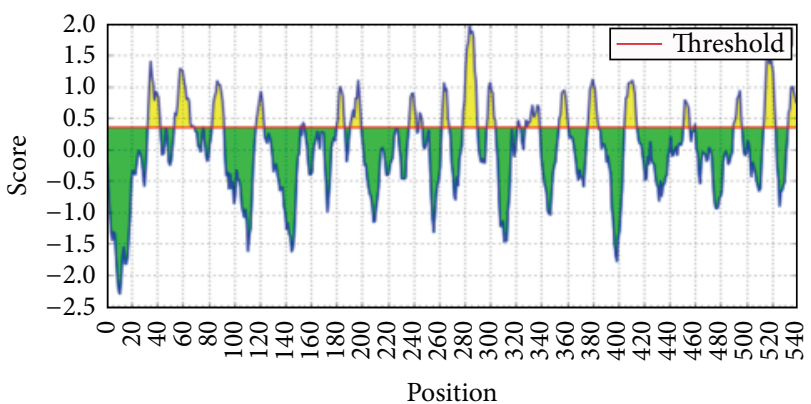

FIGURE 2: Linear B-cells epitope and their interaction with the surface membrane (yellow) in CR88 IBV strains. Note: the high east transmembrane interaction was predicted at the threshold of about 1.9 within the amino acid positions 280-290.

TABLE 3: Prediction of MHCI binding epitopes in the S1 glycoprotein of M41 IBV strains and their characteristic antigenicity scores.

\begin{tabular}{lccc}
\hline Epitope position & $\begin{array}{c}\text { Peptide } \\
\text { sequence }\end{array}$ & Conservancy \% & $\begin{array}{c}\text { Antigenicity } \\
\text { scores }\end{array}$ \\
\hline 18 & VTPLLLVTL & 98.82 & 0.8935 \\
19 & TPLLLVTLL & 9882 & 0.7935 \\
176 & VNNLTSVYL & 98.82 & 0.6733 \\
192 & SNETTDVTS & 82.35 & 0.6575 \\
208 & GGPITYKVM & 100 & 1.3364 \\
214 & KVMREVKAL & 80.00 & 0.4479 \\
356 & WFNSLSVSI & 98.82 & 1.1016 \\
387 & AYSYGGPSL & 49.41 & 0.9013 \\
462 & NVTDSAVSY & 100 & 0.6936 \\
465 & DSAVSYNYL & 100 & 0.9786 \\
467 & AVSYNYLAD & 100 & 0.5294 \\
470 & YNYLADAGL & 96.47 & 0.5650 \\
471 & NYLADAGLA & 96.47 & 0.9234 \\
472 & YLADAGLAI & 96.47 & 1.0681 \\
513 & FVVSGGKLV & 98.82 & 0.6146 \\
\hline
\end{tabular}

Note: only epitopes with 0.4 and above scores are presented.

\subsection{Prediction of T-Cell Epitope and Conservancy Analysis}

3.3.1. Prediction of MHC-I Binding Epitopes. In M41 IBV strains, a total of $21 \mathrm{MHCI}$ peptides were predicted at different amino acid positions; however, 15 of these peptides were found to be antigenic at various conservancy rates (98.82-100\%). Three epitope peptides, GGPITYKVM ${ }^{208}$, WFNSLSVSI $^{356}$, and YLADAGLAI ${ }^{472}$ relatively have high antigenicity index $(>1.0)$ as compared to other peptides (Table 3).

In the case of CR88 IBV strains, 18 MHCI epitope binding peptides were predicted. Of these, 11 peptides were found to be antigenic with most of the epitopes having conservancy rate ranging from $52.11 \%$ to $94.37 \%$ except peptide occurring at position S69 which is conserved in only $8.45 \%$. Interestingly, WFNSLSVSL ${ }^{358}$, SYNISAASV ${ }^{88}$, and YNISAASVA ${ }^{89}$ demonstrated high antigenicity index compared to other predicted peptides (Table 4). 
TABLE 4: MHCI associated antigenic peptide predicted on the S1 glycoprotein of CR88 IBV strains.

\begin{tabular}{lccc}
\hline Epitope position & $\begin{array}{c}\text { Peptide } \\
\text { sequence }\end{array}$ & Conservancy \% & Antigenicity \\
\hline S18 & GKPLLLVTL & 69.01 & 0.9669 \\
S69 & NETNNAGSV & 8.45 & 0.5716 \\
S88 & SYNISAASV & 52.11 & 1.1186 \\
S89 & YNISAASVA & 52.11 & 1.1067 \\
S216 & KVMKEVKAL & 90.14 & 0.432 \\
S358 & WFNSLSVSL & 80.28 & 1.1441 \\
S467 & EATANYSYL & 94.37 & 0.8157 \\
S472 & YSYLADGGL & 94.37 & 0.512 \\
S473 & SYLADGGLA & 94.37 & 0.8439 \\
S474 & YLADGGLAI & 97.18 & 0.9277 \\
S479 & GLAILDTSG & 94.37 & 0.4147 \\
\hline
\end{tabular}

\section{Discussion}

The use of bioinformatic and/or in silico analyses to understand infectious diseases as well as to predict novel vaccine candidate has been recently extended to poultry $[27,28]$. Most of the epitopes responsible for virus neutralization have been mapped to be located within the first and third quarters of the linear S1 glycoprotein $[13,16]$. The present study identified novel B-cells and T-cells epitopes presence in the S1 glycoprotein of M41 and CR88 IBV strains. Predicted antigenic B-cell epitopes were found to be highly conserved and demonstrated strong transmembrane potentials which probably predicts regions of virus-cell interaction. Linear Bcells epitopes located within the S1 region have been reported to play a role in virus neutralization. Using a phage display library, Zou et al. [29] identified two neutralizing linear Bcells epitopes within the $\mathrm{S} 1$ glycoprotein.

Recent studies have shown that chicken MHCI genes are categorized into MHCI associated genes (B-F) and MHCII (B-L) associated B-G genes, probably found only in poultry. Remarkably, this study has identified 21 and 18 MHCI binding peptides located within the S1 glycoproteins of M41 and CR88 IBV strains, respectively. Chickens with MHC homozygous $\mathrm{B} 12$ and $\mathrm{B} 19$ are reported to be more susceptible to infection with IBV-Grey strains as compared with B2/B2 and B5/B5 haplotype which often resist infection [30]. Recently, Tan et al. [21] used $\mathrm{BF} 2^{*} 4, \mathrm{BF} 2^{*} 12, \mathrm{BF}{ }^{*} 15$, and $\mathrm{BF} 2^{*} 19$ chicken MHCI haplotypes to predict 21 CTL-peptide candidates in Massachusetts and Australian T strains. The study revealed that the constructed poly-CTL-epitope DNA vaccine was capable of inducing protection in $90 \%$ of the vaccinated chicken following challenge with SH1208 IBV strain. In a different study, Tian et al., 2008 [31] have identified seven T- and B-cells epitopes within the IBV S1 and S2 and NP proteins chimeric DNA vaccine derived from these epitope was found to be associated with $80 \%$ protection rate. Interestingly, the regions through which B-cells and T-cells epitope were predicted in this study correspond to the IBV receptor binding domain (RBD) which play a role in sufficient binding as well as entry of IBV viruses [32]. Similarly, epitopes peptides found within $\mathrm{RBD}$ have been reported in different study to induce neutralizing antibody response, for example, against human coronavirus and mouse hepatitis virus $[33,34]$.

\section{Conclusion}

This study predicted novel antigenic B-cells and T-cell epitopes within the S1 glycoprotein of M41 and CR88 IBV strains. The characteristic antigenicity index as well as epitope conservancy rates demonstrates potentials of the identified epitope peptides as polyvalent synthetic or DNA-based peptide vaccine for application in the control of IBV infection. The use of such vaccines will likely reduce the challenges associated with live attenuated vaccines and allow broad coverage of the target IBV strains.

\section{Competing Interests}

The authors declared no conflict of interests regarding the present publication.

\section{Acknowledgments}

The authors would like to thank the Universiti Putra Malaysia and Ministry of Science, Technology and Innovation (MOSTI), Project no. 02-01-04-SF1070 for funding the research.

\section{References}

[1] D. Cavanagh, "Nidovirales: a new order comprising Coronaviridae and Arteriviridae," Archives of Virology, vol. 142, no. 3, pp. 629-633, 1997.

[2] D. Cavanagh, "Coronavirus avian infectious bronchitis virus," Veterinary Research, vol. 38, no. 2, pp. 281-297, 2007.

[3] J. J. S. de Wit, J. K. A. Cook, and H. M. J. F. van der Heijden, "Infectious bronchitis virus variants: a review of the history, current situation and control measures," Avian Pathology, vol. 40, no. 3, pp. 223-235, 2011.

[4] W. Jia, S. P. Mondal, and S. A. Naqi, "Genetic and antigenic diversity in avian infectious bronchitis virus isolates of the 1940s," Avian Diseases, vol. 46, no. 2, pp. 437-441, 2002.

[5] I. R. Alvarado, P. Villegas, J. El-Attrache, and M. W. Jackwood, "Detection of Massachusetts and Arkansas serotypes of infectious bronchitis virus in broilers," Avian Diseases, vol. 50, no. 2, pp. 292-297, 2006.

[6] D. Parsons, M. M. Ellis, D. Cavanagh, and J. K. A. Cook, "Characterisation of an infectious bronchitis virus isolated from vaccinated broiler breeder flocks," Veterinary Record, vol. 131, no. 18, pp. 408-411, 1992.

[7] Proceedings of the Angers leres Journee De La Recherche Avicole, Centre De Congres d'Angers, 1995.

[8] S. R. Hopkins and H. W. Yoder Jr., "Reversion to virulence of chicken-passaged infectious bronchitis vaccine virus," Avian Diseases, vol. 30, no. 1, pp. 221-223, 1986.

[9] E. T. McKinley, M. W. Jackwood, D. A. Hilt et al., "Attenuated live vaccine usage affects accurate measures of virus diversity and mutation rates in avian coronavirus infectious bronchitis virus," Virus Research, vol. 158, no. 1-2, pp. 225-234, 2011. 
[10] F. Bande, S. S. Arshad, A. R. Omar, M. H. Bejo, M. S. Abubakar, and Y. Abba, "Pathogenesis and diagnostic approaches of avian infectious bronchitis," Advances in Virology, vol. 2016, Article ID 4621659, 11 pages, 2016.

[11] F. Bande, S. S. Arshad, M. H. Bejo, H. Moeini, and A. R. Omar, "Progress and challenges toward the development of vaccines against avian infectious bronchitis," Journal of Immunology Research, vol. 2015, Article ID 424860, 12 pages, 2015.

[12] Z. Guo, H. Wang, T. Yang et al., "Priming with a DNA vaccine and boosting with an inactivated vaccine enhance the immune response against infectious bronchitis virus," Journal of Virological Methods, vol. 167, no. 1, pp. 84-89, 2011.

[13] A. Kant, G. Koch, D. J. van Roozelaar, J. G. Kusters, F. A. J. Poelwijk, and B. A. M. van der Zeijst, "Location of antigenic sites defined by neutralizing monoclonal antibodies on the S1 avian infectious bronchitis virus glycopolypeptide," Journal of General Virology, vol. 73, no. 3, pp. 591-596, 1992.

[14] E. W. Collisson, J. Pei, J. Dzielawa, and S. H. Seo, "Cytotoxic T lymphocytes are critical in the control of infectious bronchitis virus in poultry," Developmental and Comparative Immunology, vol. 24, no. 2-3, pp. 187-200, 2000.

[15] F. Chen, L. Pan, J. Zhang, X. Zhou, J. Li, and W. Yu, "Alleledependent association of chicken MHC class I molecules with the invariant chain," Veterinary Immunology and Immunopathology, vol. 160, no. 3-4, pp. 273-280, 2014.

[16] M. Koch, S. Camp, T. Collen et al., "Structures of an MHC class I molecule from B21 chickens illustrate promiscuous peptide binding," Immunity, vol. 27, no. 6, pp. 885-899, 2007.

[17] H.-J. Wallny, D. Avila, L. G. Hunt et al., "Peptide motifs of the single dominantly expressed class I molecule explain the striking MHC-determined response to Rous sarcoma virus in chickens," Proceedings of the National Academy of Sciences of the United States of America, vol. 103, no. 5, pp. 1434-1439, 2006.

[18] J. Ignjatovic and L. Galli, "The S1 glycoprotein but not the $\mathrm{N}$ or $\mathrm{M}$ proteins of avian infectious bronchitis virus induces protection in vaccinated chickens," Archives of Virology, vol. 138, no. 1-2, pp. 117-134, 1994.

[19] Y. Hou, Y. Guo, C. Wu, N. Shen, Y. Jiang, and J. Wang, "Prediction and identification of T cell epitopes in the H5N1 influenza virus nucleoprotein in chicken," PLoS ONE, vol. 7, no. 6, Article ID e39344, 2012.

[20] T. Yang, H.-N. Wang, X. Wang et al., "The protective immune response against infectious bronchitis virus induced by multiepitope based peptide vaccines," Bioscience, Biotechnology and Biochemistry, vol. 73, no. 7, pp. 1500-1504, 2009.

[21] L. Tan, Y. Liao, J. Fan et al., "Prediction and identification of novel IBV S1 protein derived CTL epitopes in chicken," Vaccine, vol. 34, no. 3, pp. 380-386, 2016.

[22] M. Kearse, R. Moir, A. Wilson et al., "Geneious Basic: an integrated and extendable desktop software platform for the organization and analysis of sequence data," Bioinformatics, vol. 28, no. 12, pp. 1647-1649, 2012.

[23] Y. El-Manzalawy, D. Dobbs, and V. Honavar, "Predicting linear B-cell epitopes using string kernels," Journal of Molecular Recognition, vol. 21, no. 4, pp. 243-255, 2008.

[24] A. Krogh, B. Larsson, G. Von Heijne, and E. L. L. Sonnhammer, "Predicting transmembrane protein topology with a hidden Markov model: application to complete genomes," Journal of Molecular Biology, vol. 305, no. 3, pp. 567-580, 2001.

[25] H.-H. Bui, J. Sidney, W. Li, N. Fusseder, and A. Sette, "Development of an epitope conservancy analysis tool to facilitate the design of epitope-based diagnostics and vaccines," $B M C$ Bioinformatics, vol. 8, article 361, 2007.

[26] P. Somvanshi, V. Singh, and P. K. Seth, "In silico prediction of epitopes in virulence proteins of mycobacterium tuberculosis $\mathrm{H} 37 \mathrm{Rv}$ for diagnostic and subunit vaccine design," Journal of Proteomics \& Bioinformatics, vol. 1, no. 3, pp. 143-153, 2008.

[27] S. H. Abro, K. Ullman, S. Belák, and C. Baule, "Bioinformatics and evolutionary insight on the spike glycoprotein gene of QXlike and Massachusetts strains of infectious bronchitis virus," Virology Journal, vol. 9, article 211, 2012.

[28] A. G. Ingale and S. Goto, "Prediction of CTL epitope, in silico modeling and functional analysis of cytolethal distending toxin (CDT) protein of Campylobacter jejuni," BMC Research Notes, vol. 7, no. 1, article 92, 2014.

[29] N. Zou, J. Xia, F. Wang et al., “Two novel neutralizing antigenic epitopes of the s1 subunit protein of a QX-like avian infectious bronchitis virus strain Sczy3 as revealed using a phage display peptide library," Veterinary Immunology and Immunopathology, vol. 168, no. 1-2, pp. 49-55, 2015.

[30] G. R. Banat, S. Tkalcic, J. A. Dzielawa et al., "Association of the chicken MHC B haplotypes with resistance to avian coronavirus," Developmental \& Comparative Immunology, vol. 39, no. 4, pp. 430-437, 2013.

[31] L. Tian, H.-N. Wang, D. Lu, Y.-F. Zhang, T. Wang, and R.-M. Kang, "The immunoreactivity of a chimeric multi-epitope DNA vaccine against IBV in chickens," Biochemical and Biophysical Research Communications, vol. 377, no. 1, pp. 221-225, 2008.

[32] N. Promkuntod, R. E. W. van Eijndhoven, G. de Vrieze, A. Gröne, and M. H. Verheije, "Mapping of the receptorbinding domain and amino acids critical for attachment in the spike protein of avian coronavirus infectious bronchitis virus," Virology, vol. 448, pp. 26-32, 2014.

[33] H. Kubo, Y. K. Yamada, and F. Taguchi, "Localization of neutralizing epitopes and the receptor-binding site within the amino-terminal 330 amino acids of the murine coronavirus spike protein," Journal of Virology, vol. 68, no. 9, pp. 5403-5410, 1994.

[34] A. Bonavia, B. D. Zelus, D. E. Wentworth, P. J. Talbot, and K. V. Holmes, "Identification of a receptor-binding domain of the spike glycoprotein of human coronavirus HCoV-229E," Journal of Virology, vol. 77, no. 4, pp. 2530-2538, 2003. 

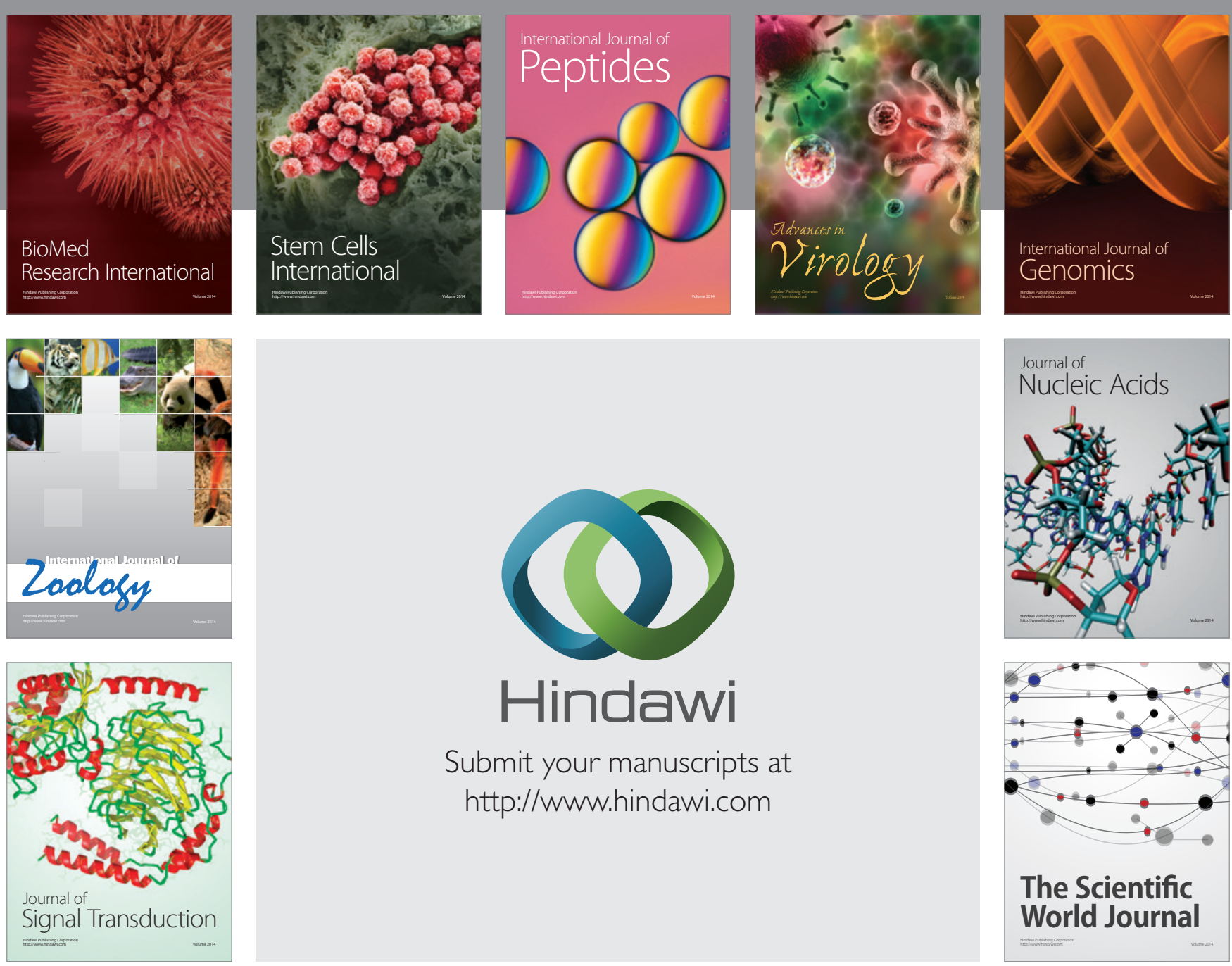

Submit your manuscripts at

http://www.hindawi.com
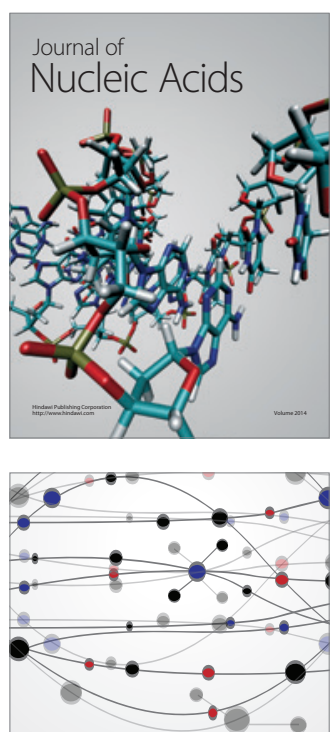

The Scientific World Journal
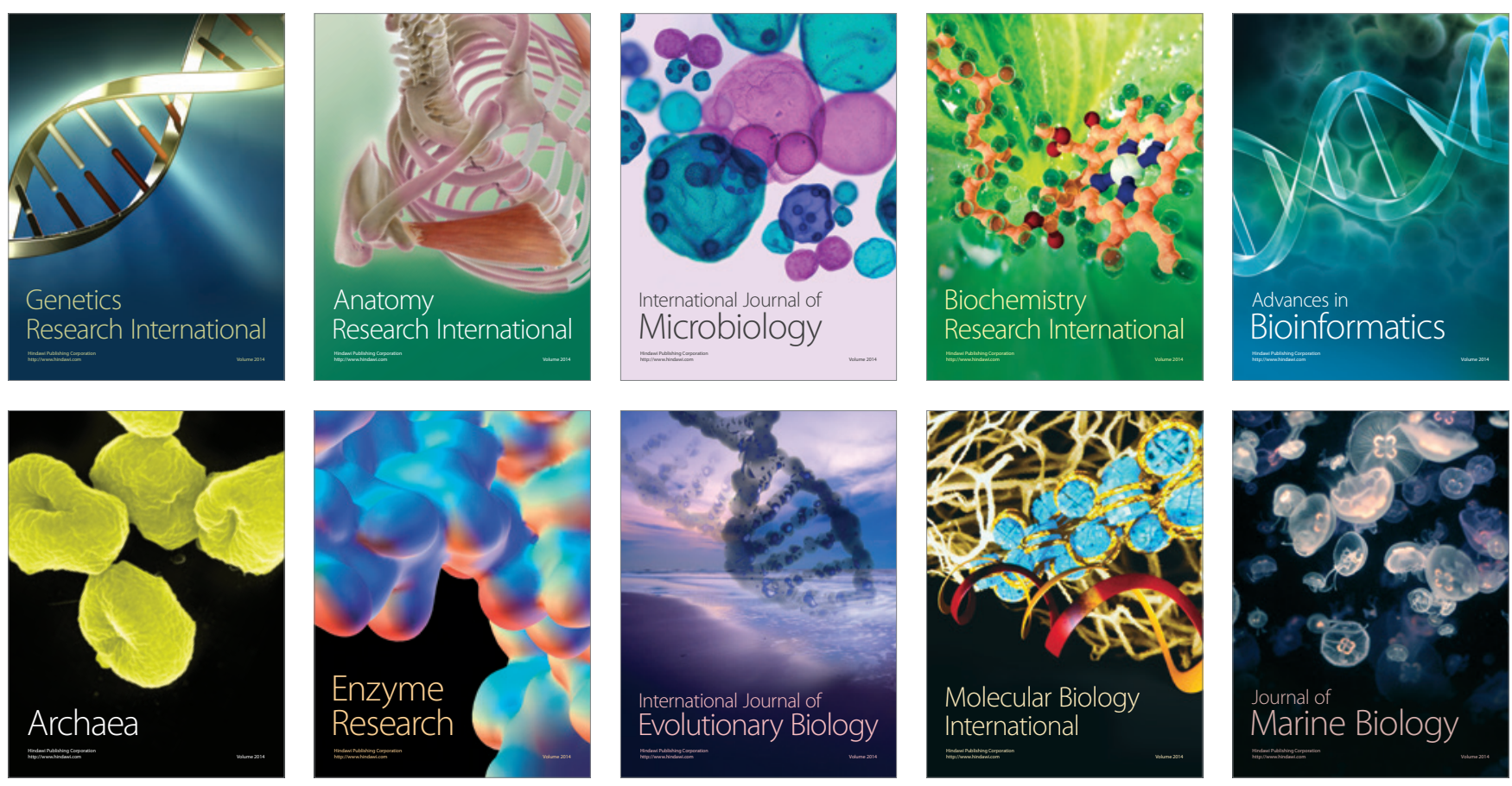